\title{
WHEN IS JONES' SPACE NORMAL?
}

\author{
WILLIAM G. FLEISSNER
}

ABSTRACT. In the search for nonmetrizable normal Moore spaces, Jones proposed the space discussed in this paper. He was unable to determine if it was normal. We show that the normality of this space depends on set theoretic principles more recent than $2^{\boldsymbol{N}_{0}}<2 \boldsymbol{K}_{1}$, which he used to show that separable normal Moore spaces are metrizable.

In $\$ 1$ we state the required set theory. In $\$ 2$ we describe a special Aronszajn tree $T$, the set of points of type $\omega$ in the Jones' road space. In $\$ 3$ we show $T$ is not normal; in $\$ 4$ we show $T$ is normal.

1. Set theory. The material here, except for $\theta$, can be found in I. Juhász' book [2]. A subset of $\omega_{1}$ is $c u b$ if it is unbounded and closed in the order topology. A subset of $\omega_{1}$ is stationary if it intersects every cub set. A function $f$ is pressing down on $x$ if for $\alpha \in x, f(\alpha)<\alpha$. An old result of Alexandrov and Urysohn is that if $f$ is pressing down on $\omega_{1}-\{0\}$, then there is some $a$ so that $f^{-1}\{\alpha\}$ is uncountable. A strengthening of this is the pressing down lemma (PDL): If $f$ is pressing down on a stationary set, it is constant on a stationary set.

Jensen's $\theta$ asserts the existence of a function $\Gamma$ such that $\Gamma(\alpha): \alpha \rightarrow \alpha$ and for all $f: \omega_{1} \rightarrow \omega_{1},\{\alpha: f \uparrow \alpha=\Gamma(\alpha)\}$ is stationary. Two elements $f$ and $g$ of a partially ordered set $\langle P, \leq\rangle$ are incompatible if there is no $h \in P$ such that $h \leq f$ and $h \leq g$. A partial order is ccc if every set of (pairwise) incompatible elements is countable; or equivalently, every uncountable $W \subset P$ contains two compatible elements. Martin's axiom (MA) states that for every ccc partial order $\langle P, \leq\rangle$ and every collection $F=$ $\left\{D_{a}: a<\omega_{1}\right\}$ of dense subsets of $P$ there is F-generic $G \subset P$; that is $G$ satisfies

(i) $x \geq y, y \in G$ implies $x \in G$;

(ii) $x, y \in G$ implies there is $z \in G, z \leq x, z \leq y$;

(iii) $G \cap D_{\alpha} \neq \varnothing$ for all $\alpha<\omega_{1}$.

Received by the editors January 15, 1974.

AMS(MOS) subject classifications (1970). Primary 02K 35, 54D15, 54E30.

Key words and phrases. Normal, normal Moore space, Aronszajn tree, Jones' road space, Martin's axiom, Jensen's principle diamond. 
MA and $D$ are (separately) consistent with the usual axioms of set theory; they were originally used to show the consistency and independence of Souslin's hypothesis.

$\omega_{1} \rightarrow\left(\omega_{1}, \omega\right)$ is a theorem of Erdös which says that if $R$ maps the unordered pairs of distinct elements of an uncountable set $W$ to $\{0,1\}$, then either there is an uncountable set $S_{0}$ which is homogeneous for 0 , i.e. such that $\alpha, \beta \in S_{0}$ implies $R(\{\alpha, \beta\})=0$, or there is an infinite set $S_{1}$ homogeneous for 1 .

A family of sets $\left\{A_{a}: \alpha<\kappa\right\}$ is called quasidisjoint (or a $\Lambda$-system) if, $A_{\alpha} \cap A_{\beta}=\bigcap\left\{A_{\alpha}: \alpha<\kappa\right\}$ for distinct $\alpha, \beta$. A popular combinatorial lemma states that if $W$ is an uncountable set of finite sets, there is an uncountable subset of $W$ of quasidisjoint sets.

2. The space $T$. If $\sigma$ is a countable, increasing, continuous sequence of rationals with a last element, let $\sigma^{*}$ be the last element, $|\sigma|$ the order type of $\sigma$, and $\sigma \uparrow \beta+1$ the sequence of the first $\beta+1$ elements of $\sigma$. A special Aronszajn tree $T$ is a collection of such sequences so that

(i) $T_{a}=\{\sigma:|\sigma|=\alpha+1\}$ is countable;

(ii) if $\sigma \in T_{a}$, then $\sigma \uparrow \beta+1 \in T_{\beta}$;

(iii) if $\sigma \in T_{\alpha}, q>\sigma^{*}$, and $\beta>\alpha$, then there is $\tau \in T_{\beta}$ so that $\tau^{*}=q$ and $\tau \uparrow \alpha+1=\sigma$.

Such a $T$ can be constructed, level by level, by transfinite induction using (iii) as induction hypothesis. The ${ }^{*}$ map order embeds $T$ into the rationals.

For $\alpha<|\sigma|$, set $V_{(\sigma, a)}=\{\tau: \tau=\sigma \mid \beta+1$ for some $\beta \geq \alpha+1\}$. Let $\beta_{\sigma}=\left\{V_{\sigma, \alpha}: \alpha<|\sigma|\right\}$ be a base for $\sigma$. This defines a regular space, since the base sets are closed. A development can be defined, using the ${ }^{*}$ map and the countableness of $Q$. If we connect $\tau \in T_{a+1}$ with $\tau \uparrow \alpha+1 \in T_{a}$ by an arc, we obtain Jones' road space [1].

Let $A$ be a subset of $T$ so that $T_{\alpha} \cap A=\left\{a_{\alpha}\right\}$ and $a_{\alpha}^{*}=1$. Then $A$ is closed and discrete. If $f$ is pressing down, let $f^{\#}(\alpha)=V_{\left(a_{\alpha}, f(\alpha)\right)^{*}}$ Placing an open set about each $a \in A$ determines a pressing down $f$, so by PDL, uncountably many open sets reach down to the same $T_{a}$, a countable set. We conclude that the open sets are not disjoint, $T$ is not collectionwise Hausdorff, and $T$ is not metrizable.

3. $\left\langle\right.$ implies $T$ is not normal. Let $A_{a}=\left\{a_{\beta}: \beta<\alpha\right\}$. For $x \subset A$, let $W(f, x)=$ closure $\bigcup\left\{f^{\#}(\alpha): a_{\alpha} \in x\right\}$. If $a_{\alpha} \notin W\left(f, A_{\alpha}\right)$, define $g(\alpha) \geq f(\alpha)$ so that $g^{\sharp}(\alpha) \cap W\left(f, A_{\alpha}\right)=\varnothing$. Then $g^{-1}\{\alpha\}$ is always countable, so by 
PDL, dom $g$ is not stationary. We conclude that $\left\{\alpha: a_{\alpha} \in W\left(f, A_{\alpha}\right)\right\}$ contains a cub set.

Let $\Gamma$ witness $\diamond$. We define inductively subsets $H_{\alpha}$ of $A_{\alpha}$ so that $H=\bigcup\left\{H_{a}: \alpha<\omega_{1}\right\}$ cannot be separated from $A-H$. Let $H_{0}=\varnothing$ and let $H_{\lambda}=\bigcup\left\{H_{\alpha}: \alpha<\lambda\right\}$ for limit $\lambda$. If $a_{\alpha} \in W\left(\Lambda^{\top}(\alpha), H_{\alpha}\right)$, let $H_{a+1}=H_{\alpha}$; otherwise let $H_{a+1}=H_{\alpha} \cup\left\{a_{a}\right\}$. If $T$ is normal there is an $f$ so that $W(f, H) \cap$ $W(f, A-H)=\varnothing$. By $\diamond$ and the preceding paragraph, there is an $\alpha$ so that $\Gamma(\alpha)=f \uparrow \alpha$ and $a_{a} \in W\left(f, A_{\alpha}\right)$. If $a_{\alpha} \in W\left(f, H_{a}\right)$, then $a_{\alpha} \in A-H$; if not, then $a_{\alpha} \in W\left(f, A_{\alpha}-H_{\alpha}\right)$ and $a_{\alpha} \in H$. So $a_{\alpha} \in W(f, H) \cap W(f, A-H)$. Contradiction.

By a similar argument, the author has shown that Gödel's axiom of constructibility implies that every normal space of character $\kappa_{1}$ is collectionwise Hausdorff [3]. Another application of $\nabla$ is to construct Aronszajn trees order embeddable in the reals but not the rationals [ 5 ].

4. MA implies $T$ is normal. Let $H, K$ be disjoint closed sets. In what follows $h$ is implicitly an element of $H$, and $k$ of $K$. Set $P=\{f: f$ is finite, $\operatorname{dom} f \subset H \cup K, f(\sigma) \in B_{\sigma}$, and $\left.f(h) \cap f(k)=\varnothing\right\}$, and $f \leq g$ if $\operatorname{dom} g \subset \operatorname{dom} f$ and for all $\sigma \in \operatorname{dom} g, g(\sigma) \subset f(\sigma)$. Then $f$ and $g$ are incompatible (have no common extension) iff for some $h, k, f(h) \cap g(k) \neq \varnothing$ or $f(k) \cap g(h) \neq \varnothing$. $D_{\sigma}=\{f: \sigma \in \operatorname{dom} f\}$ is dense. If $G$ is a $\left\{D_{\sigma}: \sigma \in H \cup K\right\}$-generic subset of $P$ then $\bigcup\{V: V=f(h)$ for some $f \in G, h \in H\}$, and $\bigcup\{V: V=f(K)$ for some $f \in G, k \in K\}$ are disjoint open sets separating $H$ and $K$. We may conclude from MA that there is such a $G$ after we show $\langle P, \leq\rangle$ has ccc.

Define $f^{*}(\sigma)$ to be $\tau^{*}$ where $\tau$ is the first initial segment of $\sigma$ in $f(\sigma)$. Arbitrarily order the domain of each $f$. Let $h_{i}^{f}$ be the $i$ th element of dom $f \cap H$, and $k_{j}^{f}$ the $j$ th element of $\operatorname{dom} f \cap K$. To simplify notation we suppress the superscript in $f\left(h_{i}\right)$.

Let $W$ be an uncountable subset of $P$. Because there are only countably many pairs of integers, and countably many finite sequences of rationals, there are integers $n, m$, and an uncountable subset $W^{\prime}$ of $W$ such that $\operatorname{dom} f=\left\{h_{0} \cdots h_{n-1}, k_{0} \cdots k_{m-1}\right\}, f^{*}\left(h_{i}\right)=g^{*}\left(h_{i}\right)$, and $f^{*}\left(k_{j}\right)=g^{*}\left(k_{j}\right)$ for all $f, g \in W^{\prime}, i<n, j<m$.

It cannot happen that simultaneously $f_{1}\left(h_{i}\right) \cap f_{2}\left(k_{j}\right) \neq \varnothing, f_{1}\left(h_{i}\right) \cap$ $f_{3}\left(k_{j}\right) \neq \varnothing$, and $f_{2}\left(h_{i}\right) \cap f_{3}\left(k_{j}\right) \neq \varnothing$. For then there would be a sequence ending in the greater of $f^{*}\left(h_{i}\right)$ and $f^{*}\left(k_{j}\right)$ in each of these intersections. Since a sequence in $T$ has only one such initial segment, we conclude that it is the same $\sigma$ in each intersection, so $\sigma \in f_{2}\left(h_{i}\right) \cap f_{2}\left(k_{j}\right)$, and $f_{2} \notin P$. 
Let $W^{\prime}=\left\{f_{\alpha}: \alpha<\omega_{1}\right\}$, and define (for $\left.\alpha<\beta\right) R_{i j}(\{\alpha, \beta\})=1$ if $f_{\alpha}\left(h_{i}\right) \cap f_{\beta}\left(k_{j}\right) \neq \varnothing$ and 0 otherwise. Define $R_{i j}^{\prime}(\{\alpha, \beta\})=1$ if $f_{\beta}\left(h_{i}\right) \cap$ $f_{\alpha}\left(k_{j}\right) \neq \varnothing$ and 0 otherwise. Apply $\omega_{1} \rightarrow\left(\omega_{1}, \omega\right)$ to the partition $R_{11}$.

By the preceding paragraph, there is no set of 3 elements homogeneous for 1 , so there is $W^{\prime \prime}$ homogeneous for 0 . Apply $\omega_{1} \rightarrow\left(\omega_{1}, \omega\right)$ to the partition $R_{12}$ restricted to $W^{\prime \prime}$. Continue in this way through all $2 m n$ partitions. The result is an uncountable set of compatible elements. We conclude $\langle P, \leq\rangle$ has ccc and that $T$ is normal.

A similar application of MA is to show that "every Aronszajn tree is embeddable in the rationals" [6]. Jensen [4] used iterated Souslin forcing to construct a model of the continuum hypothesis + "every Aronszajn tree is embeddable in the rationals." It would be nice to extend this to include "Jones' space is normal".

\section{BIBLIOGRAPHY}

1. F. Jones, Remarks on the normal Moore space metrization problem, Wisconsin Topology Seminar, 1965.

2. I. Juhász, Cardinal functions in topology, Math. Center Tracts, Amsterdam, 1971.

3. W. Fleissner, Normal Moore spaces in the constructible universe, Proc. Amer. Math. Soc. 46 (1974), 294-298.

4. R. Jensen, The consistency of Souslin's hypothesis with CH, $150 \mathrm{~h}$ andwritten pages!

5. K. Devlin, Note on a theorem of Baumgartner, Fund. Math. 76 (1972), 255260.

6. J. Baumgartner, J. Malitz and W. Reinhart, Embedding trees in the rationals, Proc. Nat. Acad. Sci. U.S.A. 67 (1970), 1748-1753.

DEPARTMENT OF MATHEMATICS, UNIVERSITY OF WISCONSIN, MADISON, WISCONSIN 53706

Current address: Department of Mathematics, McGill University, Montreal, Quebec, Canada 